$\sqrt{3}$

J. Bio-Sci. 24: 19-32, 2016

ISSN 1023-8654

http://www.banglajol.info/index.php/JBS/index

\title{
EVALUATION OF SOMACLONAL VARIANTS UNDER FIELD CONDITION FOR THE VARIETAL IMPROVEMENT OF STRAWBERRY (FRAGARIA $\times$ ANANASSA DUCH.) IN BANGLADESH
}

\author{
T Ara ${ }^{1,3^{*}}$, S Ogita ${ }^{3}$, MM Hossain $^{1,2}$ and AKMR Islam ${ }^{1}$ \\ ${ }^{1}$ Department of Botany, University of Rajshahi, Bangladesh \\ 2Institute of Biological Sciences (IBSC), University of Rajshahi, Bangladesh \\ ${ }^{3}$ Graduate School of Comprehensive Scientific Research, Prefectural University of Hiroshima, Japan
}

\begin{abstract}
The present study was conducted to standardize a suitable protocol for varietal improvement of strawberry through somaclonal variation using in vitro techniques. Leaf segments of seven strawberry varieties viz. AOG, JP-2. JP-3, Camarosa, Sweet Charly, Giant Mountain and Festival were used for callus induction and shoot regeneration. Regenerated plantlets were planted into the field to evaluate their morphological characters, yield contributing characters, response to fungal diseases and summer overcoming potentiality (\%) of the transplanted plantlets compare with their parents and data were recorded. Among the seven strawberry varieties AOG was found to be the most responsive genotype for callus induction, shoot regeneration and rooting. A total of 40-45 somaclonal variants from each of the tested varieties were established and maintained in the field and were considered as $R_{0}$ plants. There were no somaclones found resistance to fungal diseases but someone's, specially AOG SC 3 showed better tolerance than the donor plants. Comparing with donor plants and other somaclones AOG SC 3 was found better summer overcoming potentiality. It can be acceptable commercially if the good characters exhibited are transmitted through generations or could be used in future breeding programme for the improvement of strawberry varieties in Bangladesh.
\end{abstract}

Key words: Abiotic and biotic stress, callus induction, somaclonal variation, strawberry

\section{Introduction}

Strawberry cultivars are progressively studied and cultivated for its great amount of useful phytonutrients including antioxidants and phytochemicals which are advantageous in reducing the risk of tumorigenesis and heart diseases (Abbas et al. 2017). Strawberry is popular among growers of Bangladesh who get high return on their investments due to its short growing season. There are two main types of strawberry cultivars: short day or June bearing and ever bearing. Temperature may interact with photoperiods in all types of strawberries. Basically, cool temperatures promote and hot temperatures inhibit flowering (Rieger 2006). Short- day cultivars are highly sensitive to temperature. Climatic conditions of Bangladesh in winter, specifically from November to March, seem to be suitable for commercial cultivation of strawberry. There are many strawberry genotypes grown in tropical and sub-tropical environment but fruit of these genotypes are mostly unpalatable (Karim et al. 2015). Recent media reports showed that imported plants were grown well producing flowers and fruits up to March in Bangladesh. During summer and humid rainy season, almost all of the imported plants are perished due to different diseases. Plant tissue culture tools have been used to improve the accessibility of existing germplasm and to create new variations for crop improvement through

*Author for correspondence : tanziman@yahoo.com 
micro-propagation, anther culture, in vitro selection, embryo rescue, somaclonal variation, somatic hybridization and genetic transformation. Plants regenerated from calli exhibits great genetic variability in agronomic traits that known as somaclonal variation (Larkin and Scowcroft 1981). Somaclonal variations can enlarged the possibility to create genetic variation in crop plant; mainly plant characters can be altered including plant height, yield, number of flowers/plant, early flowering, grain quality, resistance to diseases, insect and pests, cold, drought and salt (Jain et al. 1998, Patnaik et al. 1999). It has been observed that strawberry cultivation in Bangladesh is highly affected with different diseases and environmental factors (Hossain 2007). Among the different diseases verticillium wilt and crown rot were found to be very prominent and high temperature (above $35^{\circ} \mathrm{C}$ ) affects strawberry cultivation in Bangladesh. In the present study, we focused on the varietal improvement of strawberry through somaclonal variation using in vitro techniques and evaluate different morphological, yield contributing characters, fungal disease incidence and summer overcoming capacity of the resulted variants.

\section{Materials and Methods}

\section{Plant materials and explants collection}

The experiment was carried out at the Plant Breeding and Gene Engineering Laboratory, Department of Botany, University of Rajshahi, Bangladesh in the year 2012. In vitro grown leaves of strawberry were used as experimental material for callus induction. Leaf segments were collected from in vitro grown plantlets maintained in mentioned Laboratory. Three Japanese (AOG, JP-2 and JP-3) and four North American varieties (Camarosa, Sweet Charly, Giant Mountain and Festival) were tested.

\section{Surface sterilization of explants}

Explants were surface sterilized with the help of savlon (ACl Pharma, Bangladesh), Tween-80 and $0.1 \%$ Mercuric chloride $\left(\mathrm{HgCl}_{2}\right)$. To ensure aseptic condition under in vitro, all instruments, glassware's and culture media were sterilized by autoclaving with $15 \mathrm{lbs} / \mathrm{sq}$. inch $\left(1.16 \mathrm{~kg} / \mathrm{cm}^{2}\right)$ pressure at $121^{\circ} \mathrm{C}$ temperature for 20 minutes.

\section{Placement of explants on culture media for callus induction}

Sterilized leaves put onto the semisolid MS (Murashige and Skoog 1962) media with different concentrations of NAA (a-napthalene acetic acid) and 2,4-D (2,4-dichlorophenoxy acetic acid) alone or in combination with BA (6-Benzyladenine). The $\mathrm{pH}$ of all media were adjusted to 5.7 before addition of agar and sterilized by autoclaving. The culture vials containing explants were placed under dark condition in a room with controlled environment (temperature $25 \pm 2{ }^{\circ} \mathrm{C}$ and humidity $50 \%$ and $16 \mathrm{~h}$ light/day by white florescent tube lights).

\section{Callus culture and shoot regeneration}

After the induction phase, the largest callus initiated from the leaves was sub cultured on to same medium. During callus culture, percentage (\%) of explants induced callus, the degree of callus development, the callus colour and nature were recorded. Then the selected calli were placed on medium supplemented with various concentration and combinations of PGRs for shoot regeneration. The percentage of calli producing shoots and total number of shoots/callus were counted in each treatment. The shoots from selected calli were excised and transferred to multiplication medium (MS $+1.5 \mathrm{mg} / \mathrm{l} \mathrm{BA}+0.5 \mathrm{mg} / \mathrm{l} \mathrm{KIN}$ ) for further growth (Ara et al. 2013). 


\section{Rooting and acclimatization}

When the regenerated shoot apices reached 4-5 cm in length with 5-6 well developed leaves, they were rescued from the culture vessels and separated from each other and cultured individually in test tubes containing 15-20 ml MS and 1/2MS with or without different combinations of auxins for root induction. Rooted plantlets were gradually acclimatized and were successfully established in the field. Prior to transfer to the field, the culture tube caps were removed and open culture vessels were kept inside the growth chamber. Then, they were taken out from the controlled environment and kept in room temperature to bring them in contact with the normal temperature for acclimatization. After that, a total of 600 plantlets were brought out of the culture vessels carefully and washed thoroughly under running tap water to make it agar gel free then transferred to plastic pots and kept under shady place and covered with polythene sheet to maintain high humidity around the juvenile plants. Finally they were transferred to the field.

\section{Field evaluation and data analysis of variants}

Data on eight morphological characters (plant height, number of leaves/ plant, petiole length, number of nodes/stolon, stolon length in $\mathrm{cm}$, number of crowns/plant, canopy size) and eight fruit yield and yield contributing characters (days to flowering, number of flower clusters/plant, number of flowers/ plant, number of fruits/plant, days to fruit harvest, average fruit weight $(\mathrm{g})$, fruit weight/plant (g) were recorded after 1, 2 and 4 months of transplantation. The wide ranges of variations were recorded. A total of 40-45 somaclones (SC) from each of the tested varieties were established and maintained in the field and were considered as Ro plants. From $\mathrm{R}_{0}$ plants four somaclones from $A O G$ and two from other six varieties were selected. Data were collected from 10 randomly selected plants and different morphological and agronomical characters were recorded and different statistical analysis (Mean \pm SE, Analysis of variance, LSD and CV\%) were done. Identification of the diseases found in the field was conducted by Plant Pathology and Microbiology Laboratory, Department of Botany, University of Rajshahi, Rajshahi, Bangladesh. Summer overcoming potentiality (\%) was measured when naturally temperature raised more than $35^{\circ} \mathrm{C}$. Tolerant to fungal diseases and summer overcoming potentiality (\%) of the transplanted plantlets were recorded.

\section{Results and Discussion}

\section{Callus induction and shoot regeneration}

Callus induction can be controlled by the level of plant growth regulators (auxin and cytokinins) in the culture medium. Leaf segments from the in vitro grown plants were used to induce callus supplemented with either 2, 4-D or NAA alone or in combination with BA. The cultured leaf explants were induced to develop callus in most of media formulations but degree of effectiveness of callusing of these formulations were different. Among the different PGR formulations, MS medium supplemented with $0.5 \mathrm{mg} / \mathrm{l} \mathrm{NAA}$ with $1.5 \mathrm{mg} / \mathrm{BA}$ was found to be the most effective media formulation in terms of percentage (\%) of explants induced to develop callus and degree of callus development. The media with 2, 4-D and NAA alone at $2.0-2.5 \mathrm{mg} / \mathrm{l}$ were also found effective PGR formulation for callus development from different parts of strawberry plants. To generate somaclonal variability, induction, maintenance and regeneration of calli are prerequisites because of various abnormalities that occur in genetic constituent during callus culture in artificial conditions are ultimately exhibited in the regenerated plants (Larkin and Scowcroft 1981, Nasrin et al. 2003, Karim et al. 2015). It has been observed in the previous studies that leaf tissue has been studied and shown to have the greatest regeneration capacity of strawberry plant tissues (Jones et al. 1988, Liu and Sanford 1988, Nehra and Stushnoff 1989, Nehra et al. 1990, Jelenkovic et al. 1991, Popescu et al. 1997, Passey et al. 2003). In addition, leaf derived callus produces more shoots than node and root (Popescu et al. 1997). In this investigation, the calli developed from leaf segments in different culture media formulations were sub- 
cultured on to regeneration medium (MS formulation) supplemented with different concentration and combination of BA and NAA and the cultures were incubated in light $(16 \mathrm{~h})$. Among the different combinations, the highest response to shoot regeneration was noticed in media contained $1.5 \mathrm{mg} / \mathrm{l} \mathrm{BA}$ and $0.5 \mathrm{mg} / \mathrm{l} \mathrm{NAA}$ (Table 1 and Fig. 1, D-E).

Table 1. Effect of different concentrations and combination of 2,4-D, NAA and BA in MS medium on callus induction and shoot regeneration from in vitro grown leaf explants. Data were recorded after four weeks incubation in dark for callus induction and five weeks of subculture for shoot regeneration.

\begin{tabular}{|c|c|c|c|c|c|c|c|c|c|c|c|c|c|c|}
\hline \multirow{3}{*}{$\begin{array}{l}\text { Growth regulators } \\
(\mathrm{mg} / \mathrm{l})\end{array}$} & \multicolumn{14}{|c|}{ Callus induction } \\
\hline & \multicolumn{7}{|c|}{$\%$ of explants induced callus } & \multicolumn{7}{|c|}{ Adventitious shoot formation } \\
\hline & 1 & 2 & 3 & 4 & 5 & 6 & 7 & 1 & 2 & 3 & 4 & 5 & 6 & 7 \\
\hline \multicolumn{15}{|l|}{ 2,4-D } \\
\hline 1.0 & 30 & 30 & 30 & 30 & 30 & 30 & 30 & - & - & - & - & - & - & - \\
\hline 2.0 & 90 & 81 & 81 & 85 & 85 & 82 & 83 & - & - & - & - & - & - & - \\
\hline 2.5 & 60 & 60 & 60 & 60 & 70 & 60 & 60 & - & - & - & - & - & - & - \\
\hline \multicolumn{15}{|l|}{ NAA } \\
\hline 1.0 & 40 & 30 & 30 & 35 & 40 & 35 & 35 & - & - & - & - & - & - & - \\
\hline 2.0 & 90 & 86 & 86 & 90 & 90 & 90 & 90 & - & - & - & - & - & - & - \\
\hline 2.5 & 70 & 70 & 70 & 70 & 70 & 75 & 65 & - & - & - & - & - & - & - \\
\hline \multicolumn{15}{|l|}{$N A A+B A$} \\
\hline $0.5+0.5$ & 30 & 10 & 30 & 20 & 20 & 30 & 20 & - & - & - & - & - & - & - \\
\hline $0.5+1.0$ & 40 & 30 & 30 & 30 & 20 & 30 & 20 & - & - & - & - & - & - & - \\
\hline $1.0+0.5$ & 55 & 55 & 55 & 40 & 40 & 40 & 40 & - & - & - & - & - & - & - \\
\hline $1.0+1.0$ & 60 & 50 & 50 & 50 & 50 & 50 & 40 & - & - & - & - & - & - & - \\
\hline $0.5+1.5$ & 90 & 90 & 90 & 90 & 90 & 90 & 90 & 3 & - & 2 & 2 & 2 & 2 & 2 \\
\hline \multicolumn{15}{|l|}{$2,4-D+B A$} \\
\hline $3.0+1.0$ & 90 & 90 & 90 & 90 & 90 & 90 & 90 & - & - & - & - & - & - & - \\
\hline $3.0+1.5$ & 70 & 80 & 80 & 80 & 80 & 80 & 80 & - & - & - & - & - & - & - \\
\hline $4.0+1.0$ & 90 & 80 & 80 & 80 & 80 & 80 & 80 & - & - & - & - & - & - & - \\
\hline $4.0+1.5$ & 80 & 80 & 80 & 80 & 80 & 80 & 80 & - & - & - & - & - & - & - \\
\hline $5.0+1.0$ & 70 & 50 & 50 & 50 & 50 & 50 & 50 & - & - & - & - & - & - & - \\
\hline
\end{tabular}


Table 1 Contd.

\begin{tabular}{|c|c|c|c|c|c|c|c|c|c|c|c|c|c|c|c|}
\hline \multirow{4}{*}{$\begin{array}{c}\text { PGR } \\
\text { supplements in } \\
\text { callus induction } \\
\text { medium (mg/l) }\end{array}$} & \multirow{4}{*}{$\begin{array}{l}\text { BA } \\
\text { supplements } \\
\text { in shoot } \\
\text { regeneration } \\
\text { medium (mg/l) }\end{array}$} & \multicolumn{14}{|c|}{ Shoot regeneration } \\
\hline & & \multicolumn{14}{|c|}{ Morphological response after 5 weeks of subculture } \\
\hline & & \multicolumn{6}{|c|}{$\begin{array}{l}\text { Percentage of calli induced shoot } \\
\text { regeneration }\end{array}$} & \multicolumn{8}{|c|}{ No. of multiple shoots/callus } \\
\hline & & 1 & 2 & 3 & 4 & 5 & 6 & 7 & 1 & 2 & 3 & 4 & 5 & 6 & 7 \\
\hline 2,4-D & 0.1 & - & - & - & . & - & . & - & rata & - & - & - & - & - & . \\
\hline \multirow[t]{3}{*}{$(2.0)$} & 0.5 & - & - & - & . & - & - & - & r & - & - & - & - & - & - \\
\hline & 1.0 & - & - & - & 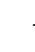 & - & . & - & . & - & - & - & - & - & - \\
\hline & 1.5 & - & - & - & . & - & . & - & . & - & - & - & - & - & - \\
\hline NAA & 0.1 & - & - & - & . & - & . & - & . & - & - & - & - & - & . \\
\hline \multirow[t]{3}{*}{ (2.0) } & 0.5 & - & - & - & 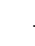 & - & . & - & . & - & - & - & - & - & . \\
\hline & 1.0 & - & - & - & . & - & . & - & . & - & - & - & - & - & . \\
\hline & 1.5 & - & - & - & 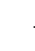 & - & 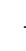 & - & . & - & - & - & - & - & - \\
\hline $2,4-D+B A$ & 0.1 & - & - & - & . & - & . & - & . & - & - & - & - & - & . \\
\hline \multirow[t]{3}{*}{$(3.0+1.0)$} & 0.5 & - & - & - & . & - & . & - & 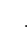 & - & - & - & - & - & . \\
\hline & 1.0 & - & - & - & . & - & 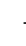 & - & . & - & - & - & - & - & . \\
\hline & 1.5 & - & - & - & 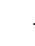 & - & . & - & 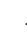 & - & - & - & - & - & 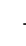 \\
\hline $\mathrm{NAA}+\mathrm{BA}$ & 0.1 & 8 & 6 & 6 & 5 & 5 & 2 & 5 & 2 & 2 & 2 & 1 & 1 & 1 & 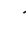 \\
\hline \multirow[t]{3}{*}{$(0.5+1.5)$} & 0.5 & 9 & 4 & 4 & 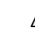 & 3 & 3 & 3 & 2 & 1 & 1 & 1 & 1 & 1 & 1 \\
\hline & 1.0 & 5 & 2 & 2 & . & - & . & - & 1 & 1 & 1 & - & - & - & 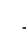 \\
\hline & 1.5 & - & - & - & 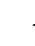 & - & . & - & . & - & - & - & - & - & . \\
\hline
\end{tabular}

- = No response, 1 = AOG, 2 = JP-2, 3 = JP-3, 4 = Camarosa, $5=$ Sweet Charly, $6=$ Giant Mountain and $7=$ Festival 


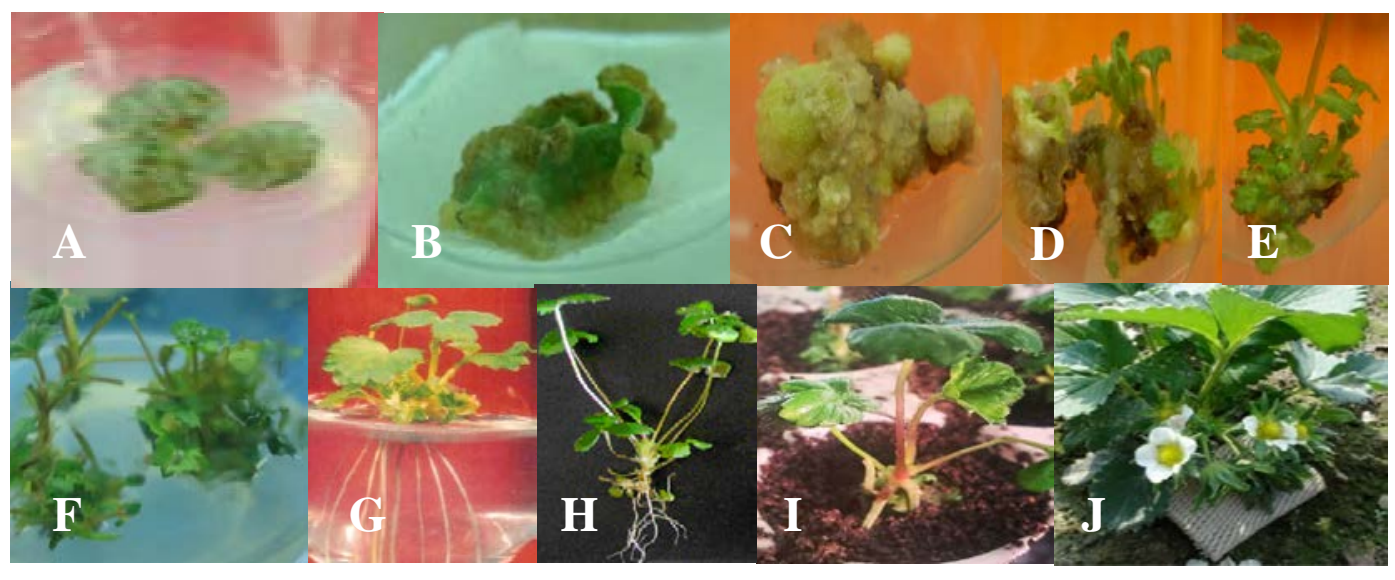

Fig. 1. Initiation of callus from in vitro grown leaf after 20 days (A-B) and callus developed after 35 days after culture in MS supplemented with $1.5 \mathrm{mg} / \mathrm{l} \mathrm{BA}+0.5 \mathrm{mg} / \mathrm{l} \mathrm{NAA}$ (C). Multiple shoots regenerated in MS medium supplemented with $0.5 \mathrm{mg} / \mathrm{l}$ BA which developed in $0.5 \mathrm{mg} / \mathrm{l} \mathrm{NAA}+1.5 \mathrm{mg} / \mathrm{l} \mathrm{BA}$ (D-E). Shoot multiplication $(F)$ and rooting $(\mathrm{G}-\mathrm{H})$ of regenerated plantlets from leaf derived calli of strawberry. Acclimatization and field establishment of rooted plantlets in plastic pots (I) and in the field with flowers (J).

The kind of PGR and the amount used is varied as the protocols for regeneration of strawberry. Nehra and Stushnoff (1989) were successful with IAA and BA, while six years later, Finstad and Martin (1995) touted the success of 2,4-D and BA. Jelenkovic et al. (1991) studied different cultivars than Nehra or Finstad, tested hypocotyls, runners, petioles, and lamina. Only young fully expanded leaves were used in the lamina study. They determined in preliminary tests that BA and 2, 4-D were the most effective PGR to use. Various combinations of $\mathrm{BA}, \mathrm{IBA}, 2,4-\mathrm{D}, \mathrm{KIN}, \mathrm{NAA}, \mathrm{TDZ}, \mathrm{CH}, \mathrm{GA}_{3}$ and $\mathrm{KNO}_{3}$ have all been reportedly used in callus induction and plant regeneration studies in strawberry (Liu and Sanford 1988, Nehra et al. 1990; Goffreda et al. 1995, Mahmoud and Kosar 2013, Rahman et al. 2015). Liu and Sanford (1988) reported using casein hydrolysate $(\mathrm{CH})$ and potassium nitrate on leaf explants of 'Allstar' strawberry. Both stimulated the production of callus and shoot and showed additive effect.

\section{Rooting and acclimatization}

The micro-shoots of strawberry inoculated in MS and $1 / 2 \mathrm{MS}$ media and plant growth regulators were induced to develop root without developing any callus at their base. When cultured in MS rooting medium without PGR, all cultured shoots developed roots within 10-15 days of inoculation, whereas $100 \%$ of the shoots induced to develop root in MS rooting medium without PGR (Table 2). Addition of auxin in rooting media accentuated rooting, but also microcuttings developed callus at their base, which hampered their field establishment. Similar results on the rooting and subsequent field establishment were also reported by Boxus (1974), Owen and Miller (1996), and Jimenez-Bermudez and Redondo-Nevado (2002). Then, the rooted plants were gradually acclimatized and transferred to the ex vitro condition for field evaluation (Fig. 1, $\mathrm{H}-\mathrm{J})$. 
Table 2. Effect of rooting media formulation on root induction of in vitro regenerated shoots of strawberry. Data were recorded after 4 weeks of culture.

\begin{tabular}{|c|c|c|c|c|c|c|c|c|c|}
\hline \multirow{2}{*}{\multicolumn{3}{|c|}{ Treatment (mg/l) }} & \multicolumn{7}{|c|}{ Shoots induced root development (\%) } \\
\hline & & & AOG & $\mathrm{JP}-2$ & $\mathrm{JP}-3$ & Camarosa & Sweet Charly & Giant Mountain & Festival \\
\hline \multirow{8}{*}{ Full strength } & \multirow{4}{*}{ NAA } & 0.1 & 70 & 65 & 66 & 70 & 65 & 69 & 67 \\
\hline & & 0.5 & 90 & 86 & 85 & 88 & 85 & 85 & 88 \\
\hline & & 1.0 & 95 & 90 & 90 & 90 & 88 & 90 & 90 \\
\hline & & 2.0 & 91 & 90 & 90 & 90 & 90 & 90 & 90 \\
\hline & \multirow{4}{*}{ IBA } & 0.1 & 60 & 60 & 58 & 60 & 60 & 58 & 60 \\
\hline & & 0.5 & 88 & 87 & 87 & 88 & 80 & 80 & 80 \\
\hline & & 1.0 & 90 & 90 & 90 & 90 & 90 & 90 & 90 \\
\hline & & 2.0 & 88 & 85 & 84 & 83 & 83 & 85 & 83 \\
\hline \multirow{8}{*}{ Half strength } & \multirow{4}{*}{ NAA } & 0.1 & 65 & 60 & 65 & 65 & 66 & 60 & 65 \\
\hline & & 0.5 & 65 & 60 & 60 & 60 & 63 & 63 & 60 \\
\hline & & 1.0 & 85 & 80 & 80 & 85 & 85 & 85 & 80 \\
\hline & & 2.0 & 55 & 55 & 56 & 57 & 56 & 55 & 56 \\
\hline & \multirow{4}{*}{ IBA } & 0.1 & 45 & 45 & 43 & 50 & 50 & 55 & 44 \\
\hline & & 0.5 & 60 & 57 & 58 & 58 & 60 & 57 & 58 \\
\hline & & 1.0 & 75 & 75 & 70 & 77 & 77 & 75 & 72 \\
\hline & & 2.0 & 60 & 60 & 59 & 60 & 58 & 60 & 60 \\
\hline \multirow{2}{*}{$\begin{array}{l}\text { Without } \\
\text { growth } \\
\text { regulators }\end{array}$} & \multicolumn{2}{|c|}{$1 / 2 \mathrm{MSO}$} & 96 & 95 & 95 & 96 & 95 & 92 & 92 \\
\hline & \multicolumn{2}{|c|}{ MSO } & 100 & 100 & 100 & 100 & 100 & 100 & 100 \\
\hline
\end{tabular}

\section{Field evaluation and data analysis}

Among the seven strawberry varieties, 30-50 somaclones from each of the varieties were transplanted to the field and considered as Ro plants. In order to evaluate the somaclonal variation among the plants, data were recorded on different morphological and agronomical charactres. It was observed that regenerated plants grown in the field were not identical to their mother plant (Fig. 2, Tables 3-4). In the present study seven strawberry genotypes and their somaclones were evaluated for sixteen characters (eight morphological and eight yield contributing characters). Collected data were analyzed in order to estimate mean with standard error, analysis of variance (ANOVA), least significant difference (LSD) and coefficient of variability (CV\%). In the analysis of variance the main item genotype was highly significant for all characters at $5 \%$ level of significance (Tables 3-4). These results indicate that genotypes were different from each other and justify their inclusion in the present investigation as materials. The replication items were non-significant for all characters. Haque (1997) obtained similar results in chickpea.

In the last part of this research, field evaluation of the seven strawberry varieties and their somaclones was conducted under different stress conditions: biotic (fungal diseases) and abiotic stress (temperature). Verticillium wilt, phytophthora crown rot, leaf scotch, leaf spot, leaf blight and botrytis fruit rot disease were found in the strawberry field (Fig. 3, A-F). 
Table 3. Morphological characters of selected somaclones and comparison with their respective seven strawberry parents.

\begin{tabular}{|c|c|c|c|c|c|c|c|c|}
\hline Varieties/ somaclones & $\begin{array}{l}\text { Plant height } \\
(\mathrm{cm}) \\
(\text { Mean } \pm \text { SE) }\end{array}$ & $\begin{array}{l}\text { No. of } \\
\text { leaves/plant } \\
\text { (Mean } \pm \text { SE) }\end{array}$ & $\begin{array}{l}\text { Petiole length } \\
(\mathrm{cm}) \\
(\text { Mean } \pm \text { SE) }\end{array}$ & $\begin{array}{l}\text { No. of stolons/ } \\
\text { Plant } \\
\text { (Mean } \pm S E \text { ) }\end{array}$ & $\begin{array}{l}\text { No. of nodes/ } \\
\text { Stolon } \\
\text { (Mean } \pm \text { SE) }\end{array}$ & $\begin{array}{l}\text { Stolon length (cr } \\
\text { (Mean } \pm \text { SE) }\end{array}$ & $\begin{array}{l}\text { No. of crowns/ } \\
\text { Plant } \\
\text { (Mean } \pm \text { SE) }\end{array}$ & $\begin{array}{l}\text { Canopy size } \\
\left(\mathrm{cm}^{2}\right) \\
(\text { Mean } \pm \mathrm{SE})\end{array}$ \\
\hline AOG & $16.40 \pm 0.21$ & $20.23 \pm 0.14$ & $14.23 \pm 0.14$ & $10.07 \pm 0.43$ & $4.27 \pm 0.14$ & $120.4 \pm 0.35$ & $3.20 \pm 0.058$ & $364.23 \pm 8.14$ \\
\hline AOG SC 1 & $21.47 \pm 0.58$ & $26.40 \pm 0.21$ & $16.07 \pm 0.26$ & $14.23 \pm 0.14$ & $4.90 \pm 0.21$ & $161.5 \pm 13.8$ & $6.50 \pm 0.057$ & $474.40 \pm 0.26$ \\
\hline AOG SC 2 & $28.00 \pm 0.29$ & $25.27 \pm 0.15$ & $15.40 \pm 0.20$ & $12.43 \pm 0.23$ & $4.40 \pm 0.15$ & $155.0 \pm 0.40$ & $5.40 \pm 0.057$ & $374.37 \pm 0.30$ \\
\hline AOG SC 3 & $31.97 \pm 0.32$ & $30.33 \pm 0.08$ & $17.23 \pm 0.14$ & $16.37 \pm 0.18$ & $6.27 \pm 0.14$ & $163.1 \pm 0.20$ & $7.50 \pm 0.057$ & $594.03 \pm 0.52$ \\
\hline AOG SC 4 & $17.40 \pm 0.21$ & $20.47 \pm 0.14$ & $15.47 \pm 0.14$ & $13.27 \pm 0.14$ & $5.27 \pm 0.14$ & $145.0 \pm 0.11$ & $5.50 \pm 0.057$ & $473.87 \pm 0.63$ \\
\hline LSD value (at $5 \%$ level) & 2.2250 & 1.3252 & 1.8361 & 2.3382 & 1.3902 & 55.2871 & 0.5272 & 31.5607 \\
\hline $\mathrm{CV} \%$ & 1.8763 & 1.0495 & 2.2758 & 3.4236 & 5.3822 & 7.2115 & 1.8233 & 1.3446 \\
\hline$J P-2$ & $15.47 \pm 0.20$ & $20.27 \pm 0.39$ & $13.57 \pm 0.23$ & $4.27 \pm 0.12$ & $2.43 \pm 0.09$ & $114.23 \pm 3.03$ & $3.27 \pm 0.14$ & $382.77 \pm 1.22$ \\
\hline JP- 2 SC 1 & $24.00 \pm 0.29$ & $22.67 \pm 0.12$ & $15.23 \pm 0.14$ & $5.33 \pm 0.12$ & $3.20 \pm 0.10$ & $145.27 \pm 0.14$ & $4.37 \pm 0.07$ & $450.27 \pm 0.15$ \\
\hline JP- 2 SC 2 & $22.23 \pm 0.21$ & $24.50 \pm 0.17$ & $15.33 \pm 0.17$ & $5.83 \pm 0.067$ & $3.30 \pm 0.11$ & $155.33 \pm 0.24$ & $6.47 \pm 0.09$ & $474.40 \pm 0.14$ \\
\hline LSD value (at $5 \%$ level) & 0.5941 & 1.3090 & 1.3006 & 0.5286 & 0.2426 & 11.6129 & 0.7123 & 4.4029 \\
\hline $\mathrm{CV} \%$ & 0.7940 & 1.6006 & 2.4299 & 2.8243 & 2.2388 & 2.3083 & 4.1657 & 0.2777 \\
\hline$J P-3$ & $16.13 \pm 0.18$ & $15.47 \pm 0.20$ & $13.27 \pm 0.15$ & $4.40 \pm 0.05$ & $2.33 \pm 0.09$ & $100.70 \pm 0.44$ & $3.03 \pm 0.26$ & $383.40 \pm 3.31$ \\
\hline JP-3 SC 1 & $25.03 \pm 0.26$ & $19.60 \pm 0.20$ & $14.23 \pm 0.15$ & $6.27 \pm 0.15$ & $3.13 \pm 0.09$ & $149.73 \pm 0.39$ & $5.40 \pm 0.06$ & $451.23 \pm 0.16$ \\
\hline JP-3 SC 2 & $22.00 \pm 0.23$ & $18.60 \pm 0.21$ & $13.80 \pm 0.15$ & $6.10 \pm 0.10$ & $4.23 \pm 0.12$ & $150.13 \pm 0.23$ & $6.63 \pm 0.09$ & $462.37 \pm 0.30$ \\
\hline LSD value (at $5 \%$ level) & 0.3536 & 1.3090 & 0.9394 & 0.7377 & 0.2101 & 1.8710 & 1.0147 & 11.6663 \\
\hline $\mathrm{CV} \%$ & 0.4616 & 2.0112 & 1.8755 & 3.6279 & 1.7856 & 0.3851 & 5.5531 & 0.7417 \\
\hline Camarosa & $15.43 \pm 0.23$ & $18.47 \pm 0.26$ & $14.30 \pm 0.15$ & $4.33 \pm 0.09$ & $2.47 \pm 0.09$ & $113.73 \pm 3.38$ & $2.80 \pm 0.06$ & $361.60 \pm 2.27$ \\
\hline Camarosa SC 1 & $22.27 \pm 0.15$ & $20.37 \pm 0.09$ & $15.27 \pm 0.15$ & $4.67 \pm 0.12$ & $3.27 \pm 0.09$ & $144.30 \pm 0.15$ & $6.17 \pm 0.09$ & $474.37 \pm 0.30$ \\
\hline Camarosa SC 2 & $24.43 \pm 0.12$ & $22.50 \pm 0.17$ & $15.27 \pm 0.15$ & $4.77 \pm 0.09$ & $3.07 \pm 0.12$ & $148.20 \pm 0.12$ & $6.30 \pm 0.06$ & $374.30 \pm 0.36$ \\
\hline LSD value (at $5 \%$ level) & 1.3174 & 1.3748 & 1.0573 & 0.6417 & 0.6643 & 12.3271 & 0.4113 & 8.8358 \\
\hline $\mathrm{CV} \%$ & 1.7483 & 1.8482 & 1.9445 & 3.8437 & 6.2241 & 2.5021 & 2.2213 & 0.6020 \\
\hline Sweet Charly & $15.33 \pm 0.17$ & $12.30 \pm 0.15$ & $14.27 \pm 0.15$ & $4.60 \pm 0.06$ & $2.47 \pm 0.09$ & $109.37 \pm 5.33$ & $2.50 \pm 0.06$ & $337.40 \pm 3.05$ \\
\hline Sweet Charly SC 1 & $22.23 \pm 0.15$ & $17.47 \pm 0.26$ & $14.57 \pm 0.18$ & $6.33 \pm 0.12$ & $4.13 \pm 0.09$ & $155.23 \pm 0.15$ & $6.17 \pm 0.12$ & $474.43 \pm 0.23$ \\
\hline Sweet Charly SC 2 & $24.03 \pm 0.29$ & $14.53 \pm 0.15$ & $15.30 \pm 0.12$ & $5.77 \pm 0.09$ & $3.30 \pm 0.12$ & $148.27 \pm 0.15$ & $5.30 \pm 0.15$ & $374.57 \pm 0.12$ \\
\hline LSD value (at $5 \%$ level) & 1.4779 & 0.6124 & 1.0362 & 0.5558 & 0.6967 & 18.8898 & 0.6752 & 11.6672 \\
\hline $\mathrm{CV} \%$ & 1.9783 & 1.1399 & 1.9360 & 2.7441 & 5.8026 & 3.7726 & 3.9865 & 0.8109 \\
\hline Giant Mountain & $16.47 \pm 0.15$ & $12.27 \pm 0.15$ & $15.20 \pm 0.15$ & $3.40 \pm 0.06$ & $2.33 \pm 0.07$ & $115.93 \pm 3.53$ & $3.30 \pm 0.12$ & $357.50 \pm 1.31$ \\
\hline Giant Mountain SC 1 & $22.30 \pm 0.15$ & $16.63 \pm 0.13$ & $16.63 \pm 0.13$ & $5.50 \pm 0.06$ & $3.00 \pm 0.06$ & $155.67 \pm 0.12$ & $6.20 \pm 0.06$ & $450.53 \pm 0.20$ \\
\hline Giant Mountain SC 2 & $22.13 \pm 0.32$ & $13.23 \pm 0.15$ & $15.50 \pm 0.29$ & $5.67 \pm 0.09$ & $3.03 \pm 0.09$ & $150.23 \pm 0.12$ & $5.70 \pm 0.06$ & $474.53 \pm 0.15$ \\
\hline LSD value (at $5 \%$ level) & 1.4247 & 1.0362 & 1.1279 & 0.4851 & 0.1918 & 12.8639 & 0.5753 & 4.5604 \\
\hline $\mathrm{CV} \%$ & 1.9294 & 2.0278 & 1.9649 & 2.7460 & 1.8898 & 2.5145 & 3.1207 & 0.2932 \\
\hline Festival & $15.57 \pm 0.23$ & $13.10 \pm 0.21$ & $13.23 \pm 0.15$ & $2.63 \pm 0.09$ & $2.30 \pm 0.06$ & $113.03 \pm 0.32$ & $2.80 \pm 0.06$ & $218.83 \pm 4.41$ \\
\hline Festival SC 1 & $25.30 \pm 0.41$ & $18.27 \pm 0.15$ & $15.20 \pm 0.15$ & $3.57 \pm 0.12$ & $2.77 \pm 0.09$ & $151.73 \pm 3.37$ & $6.23 \pm 0.19$ & $299.63 \pm 0.19$ \\
\hline Festival SC 2 & $22.53 \pm 0.14$ & $20.33 \pm 0.09$ & $15.20 \pm 0.15$ & $4.30 \pm 0.06$ & $3.20 \pm 0.06$ & $150.27 \pm 0.12$ & $5.80 \pm 0.06$ & $333.20 \pm 0.17$ \\
\hline LSD value (at $5 \%$ level) & 1.4169 & 1.0813 & 1.0573 & 0.6474 & 0.5073 & 12.4074 & 0.8489 & 16.0482 \\
\hline $\mathrm{CV} \%$ & 1.8428 & 1.7246 & 1.9980 & 5.0843 & 5.0604 & 2.4650 & 4.7191 & 1.5537 \\
\hline
\end{tabular}


Table 4. Fruit yield and yield contributing characters of selected somaclones and comparison with their respective seven strawberry parents.

\begin{tabular}{|c|c|c|c|c|c|c|c|c|}
\hline $\begin{array}{l}\text { Varieties/ } \\
\text { somaclones }\end{array}$ & $\begin{array}{l}\text { Days to } \\
\text { Flowering } \\
\text { (Mean } \pm S E)\end{array}$ & $\begin{array}{l}\text { No. of flower } \\
\text { clusters/plant } \\
\text { (Mean } \pm \text { SE) }\end{array}$ & $\begin{array}{l}\text { No. of flowers/ } \\
\text { Plant } \\
\text { (Mean } \pm \text { SE) }\end{array}$ & $\begin{array}{l}\text { No. of fruits/ } \\
\text { Cluster } \\
\text { (Mean } \pm \text { SE) }\end{array}$ & $\begin{array}{l}\text { No. of fruits/plan } \\
\text { (Mean } \pm S E)\end{array}$ & $\begin{array}{l}\text { Days to fruit } \\
\text { Harvest } \\
\text { (Mean } \pm \text { SE) }\end{array}$ & $\begin{array}{l}\text { Average fruit w } \\
(\mathrm{g}) \\
\text { (Mean } \pm \text { SE) }\end{array}$ & $\begin{array}{l}\text { Fruit wt./plant } \\
\text { (Mean } \pm \text { SE) }\end{array}$ \\
\hline AOG & $67.67 \pm 0.33$ & $6.33 \pm 0.33$ & $15.67 \pm 0.33$ & $2.67 \pm 0.33$ & $9.67 \pm 0.33$ & $90.33 \pm 0.33$ & $25.30 \pm 0.15$ & $244.33 \pm 9.67$ \\
\hline AOG SC 1 & $61.67 \pm 0.33$ & $7.67 \pm 0.33$ & $17.67 \pm 0.33$ & $4.00 \pm 0.33$ & $15.33 \pm 0.33$ & $84.67 \pm 0.33$ & $30.03 \pm 0.14$ & $460.43 \pm 8.28$ \\
\hline AOG SC 2 & $62.00 \pm 0.33$ & $6.33 \pm 0.33$ & $17.67 \pm 0.33$ & $4.33 \pm 0.57$ & $17.67 \pm 0.57$ & $84.00 \pm 0.33$ & $28.23 \pm 0.14$ & $498.80 \pm 10.04$ \\
\hline AOG SC 3 & $60.00 \pm 0.33$ & $8.67 \pm 0.33$ & $21.67 \pm 0.33$ & $6.33 \pm 0.33$ & $20.33 \pm 0.35$ & $81.67 \pm 0.33$ & $38.00 \pm 0.11$ & $772.60 \pm 10.67$ \\
\hline AOG SC 4 & $61.67 \pm 0.33$ & $7.33 \pm 0.33$ & $18.67 \pm 0.33$ & $3.67 \pm 0.33$ & $15.00 \pm 0.58$ & $82.67 \pm 0.33$ & $30.13 \pm 0.23$ & $442.73 \pm 7.99$ \\
\hline LSD value (at $5 \%$ level) & 2.3011 & 3.0440 & 3.0440 & 3.3871 & 3.7576 & 2.8954 & 1.0975 & 91.2854 \\
\hline CV\% & 0.7144 & 8.1414 & 3.2387 & 15.6733 & 4.6814 & 0.6646 & 0.7031 & 3.6672 \\
\hline JP-2 & $69.67 \pm 0.33$ & $5.67 \pm 0.33$ & $14.33 \pm 0.33$ & $2.33 \pm 0.33$ & $6.67 \pm 0.33$ & $90.33 \pm 0.33$ & $20.37 \pm 0.09$ & $144.93 \pm 0.74$ \\
\hline JP- 2 SC 1 & $61.33 \pm 0.33$ & $7.67 \pm 0.33$ & $17.67 \pm 0.33$ & $4.33 \pm 0.33$ & $14.33 \pm 0.33$ & $84.67 \pm 0.33$ & $32.17 \pm 0.73$ & $504.33 \pm 2.89$ \\
\hline JP- 2 SC 2 & $61.67 \pm 0.33$ & $6.67 \pm 0.33$ & $18.67 \pm 0.33$ & $2.67 \pm 0.33$ & $13.67 \pm 0.33$ & $84.67 \pm 0.33$ & $22.10 \pm 1.70$ & $338.17 \pm 9.27$ \\
\hline LSD value (at $5 \%$ level) & 2.4255 & 2.1006 & 2.4255 & 2.4255 & 1.9176 & 2.4255 & 5.2738 & 31.1646 \\
\hline CV\% & 1.0381 & 8.6603 & 3.9474 & 21.4286 & 4.5610 & 0.7702 & 5.8266 & 2.6024 \\
\hline$J P-3$ & $70.00 \pm 0.33$ & $6.00 \pm 0.33$ & $14.33 \pm 0.33$ & $2.00 \pm 0.33$ & $6.67 \pm 0.33$ & $92.67 \pm 0.33$ & $22.27 \pm 0.15$ & $154.57 \pm 0.43$ \\
\hline JP-3 SC 1 & $61.67 \pm 0.33$ & $7.67 \pm 0.33$ & $17.67 \pm 0.33$ & $2.67 \pm 0.33$ & $14.33 \pm 0.33$ & $83.67 \pm 1.20$ & $31.17 \pm 0.65$ & $482.27 \pm 3.05$ \\
\hline JP-3 SC 2 & $61.67 \pm 0.33$ & $6.67 \pm 0.33$ & $18.67 \pm 0.33$ & $6.00 \pm 0.57$ & $14.33 \pm 0.33$ & $84.67 \pm 0.33$ & $32.43 \pm 0.23$ & $497.03 \pm 2.57$ \\
\hline LSD value (at $5 \%$ level) & 1.9176 & 1.2128 & 2.4255 & 2.8442 & 1.2128 & 3.6383 & 1.7491 & 14.2024 \\
\hline CV\% & 0.8178 & 4.9180 & 3.9474 & 21.9863 & 2.8302 & 1.1494 & 1.6796 & 1.0328 \\
\hline Camarosa & $70.00 \pm 0.33$ & $5.33 \pm 0.33$ & $16.33 \pm 0.33$ & $2.00 \pm 0.33$ & $5.33 \pm 0.33$ & $90.33 \pm 0.33$ & $21.90 \pm 1.55$ & $132.67 \pm 5.36$ \\
\hline Camarosa SC 1 & $61.67 \pm 0.33$ & $5.67 \pm 0.33$ & $18.33 \pm 0.33$ & $3.00 \pm 0.33$ & $13.67 \pm 0.33$ & $84.33 \pm 0.67$ & $31.03 \pm 0.20$ & $438.37 \pm 1.44$ \\
\hline Camarosa SC 2 & $61.00 \pm 0.58$ & $6.67 \pm 0.33$ & $18.67 \pm 0.33$ & $6.67 \pm 0.33$ & $13.33 \pm 0.33$ & $85.00 \pm 0.33$ & $32.43 \pm 0.09$ & $426.60 \pm 2.01$ \\
\hline LSD value (at $5 \%$ level) & 1.9176 & 1.2128 & 2.4255 & 1.2128 & 2.4255 & 3.2087 & 5.7960 & 21.7791 \\
\hline CV\% & 0.8207 & 5.6604 & 3.7500 & 8.5714 & 6.1856 & 1.0189 & 5.5983 & 1.8001 \\
\hline Sweet Charly & $71.67 \pm 1.66$ & $5.33 \pm 0.33$ & $15.33 \pm 0.33$ & $2.00 \pm 0.33$ & $6.00 \pm 0.33$ & $90.33 \pm 0.33$ & $23.10 \pm 0.23$ & $144.00 \pm 1.53$ \\
\hline Sweet Charly SC 1 & $61.67 \pm 0.33$ & $6.33 \pm 0.33$ & $18.33 \pm 0.33$ & $2.67 \pm 0.33$ & $14.33 \pm 0.33$ & $84.67 \pm 0.33$ & $31.93 \pm 0.87$ & $461.33 \pm 2.62$ \\
\hline Sweet Charly SC 2 & $61.67 \pm 0.33$ & $7.00 \pm 0.58$ & $17.33 \pm 0.33$ & $3.00 \pm 0.33$ & $13.67 \pm 0.33$ & $84.33 \pm 0.33$ & $23.37 \pm 0.44$ & $334.50 \pm 0.58$ \\
\hline LSD value (at $5 \%$ level) & 7.2766 & 1.2128 & 2.1006 & 1.2128 & 1.4853 & 2.4255 & 4.0296 & 10.0375 \\
\hline CV\% & 3.0769 & 5.3571 & 3.3962 & 13.0435 & 3.6022 & 0.7712 & 4.2381 & 0.8806 \\
\hline Giant Mountain & $70.33 \pm 0.33$ & $5.67 \pm 0.33$ & $15.33 \pm 0.33$ & $2.00 \pm 0.33$ & $4.67 \pm 0.33$ & $90.33 \pm 0.33$ & $23.37 \pm 0.19$ & $122.67 \pm 3.71$ \\
\hline Giant Mountain SC 1 & $62.00 \pm 0.33$ & $6.67 \pm 0.33$ & $18.33 \pm 0.33$ & $2.67 \pm 0.33$ & $13.33 \pm 0.33$ & $84.33 \pm 0.33$ & $32.23 \pm 0.12$ & $433.80 \pm 0.31$ \\
\hline Giant Mountain SC 2 & $61.67 \pm 0.33$ & $6.33 \pm 0.33$ & $17.00 \pm 0.58$ & $3.00 \pm 0.33$ & $13.67 \pm 0.33$ & $84.67 \pm 0.33$ & $32.60 \pm 0.15$ & $463.43 \pm 1.62$ \\
\hline LSD value (at 5\% level) & 1.4853 & 2.4255 & 3.2087 & 1.2128 & 2.4255 & 2.4255 & 0.5355 & 14.4131 \\
\hline CV\% & 0.6313 & 10.7143 & 5.2219 & 13.0435 & 6.3158 & 0.7712 & 0.5007 & 1.1653 \\
\hline Festival & $75.00 \pm 0.58$ & $5.33 \pm 0.33$ & $13.00 \pm 0.58$ & $2.00 \pm 0.33$ & $5.67 \pm 0.33$ & $90.33 \pm 0.33$ & $21.67 \pm 0.07$ & $138.87 \pm 5.88$ \\
\hline Festival SC 1 & $62.00 \pm 0.58$ & $6.67 \pm 0.33$ & $16.00 \pm 0.58$ & $2.33 \pm 0.33$ & $5.67 \pm 0.33$ & $84.67 \pm 0.33$ & $30.93 \pm 0.59$ & $445.83 \pm 1.09$ \\
\hline Festival SC 2 & $61.67 \pm 0.33$ & $7.00 \pm 0.58$ & $15.67 \pm 0.33$ & $3.00 \pm 0.33$ & $12.67 \pm 0.33$ & $84.67 \pm 0.33$ & $31.83 \pm 0.87$ & $429.23 \pm 0.80$ \\
\hline LSD value (at $5 \%$ level) & 3.8351 & 2.9707 & 3.2087 & 1.2128 & 2.1006 & 2.4255 & 4.6751 & 22.4657 \\
\hline CV\% & 1.5918 & 12.8921 & 5.9233 & 13.6364 & 5.4127 & 0.7702 & 4.5656 & 1.8270 \\
\hline
\end{tabular}




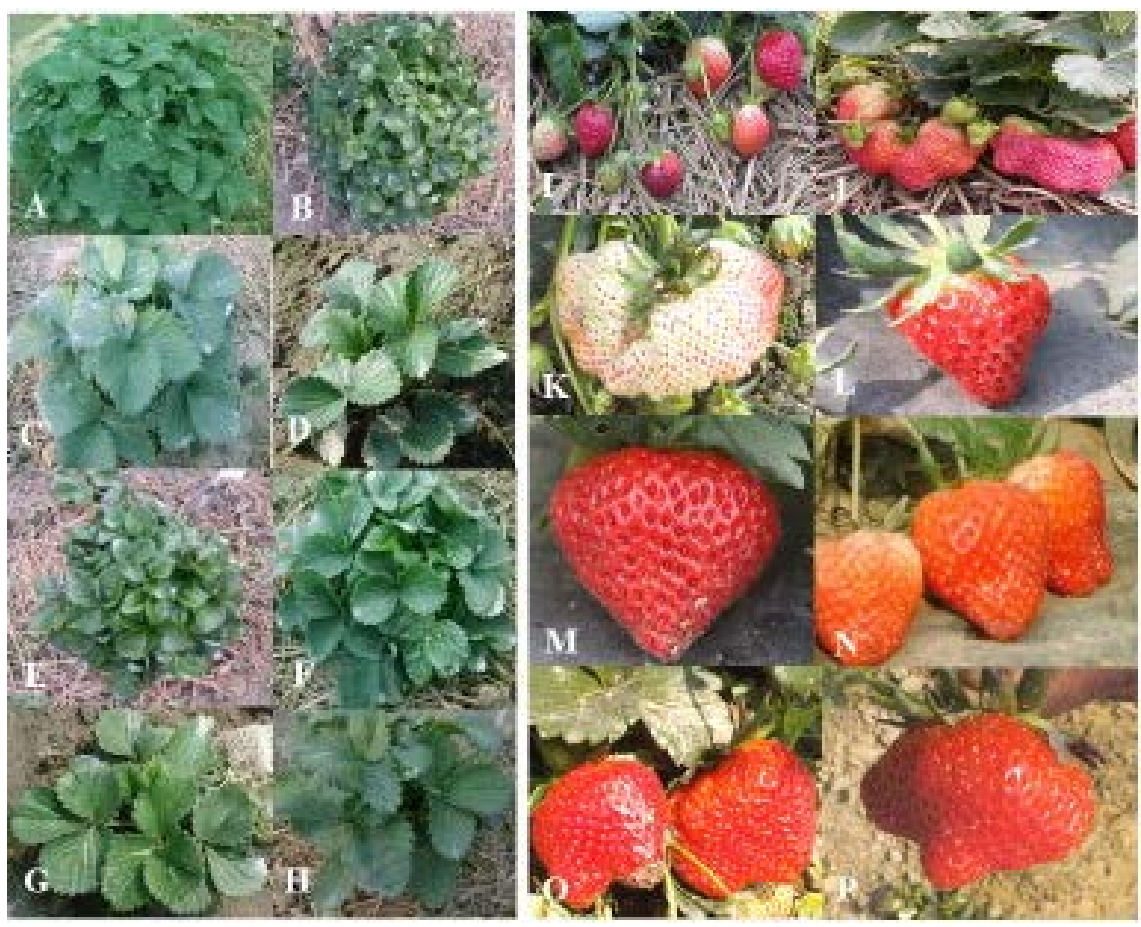

Fig. 2. Different plant types (A-H) and fruit shapes (I-P) showing somaclonal variations.

Among the six strawberry diseases, disease incidences (\%) of verticillium wilt and phytophthora crown rot was high (60\%) in seven donor parent's viz. AOG, JP-2, JP-3, Camarosa, Sweet Charly, Giant Mountain and Festival but in somaclones it was 15\% (Table 5). Other diseases viz. leaf scotch, leaf spot, leaf blight and botrytis fruit rot, disease incidence (\%) was high (45-50\%) in donor plants but in their somaclones it was low $(10 \%)$. Most of the plants were severally affected with these diseases during the summer and were perished. There were no plants found resistance to fungal diseases.

High temperature one of the major factor that affects strawberry cultivation. During the summer month AprilMay temperature becomes high (above $38^{\circ} \mathrm{C}$ ) and the plants do not perpetuate in the field. In terms of summer overcoming capacity, majority of plants were found heat sensitive in donor plants. In their somaclones, $75-80 \%$ plants showed moderate summer overcoming capacity and $15-20 \%$ plants showed high summer overcomming capacity. Somaclone AOG SC 3 showed better performance than other somaclones and donor parents in terms of summer overcoming capacity (Table 6). These somaclones can be acceptable commercially if the good characters exhibited are transmitted through generations or could be used in future breeding programme for the improvement of strawberry varieties in Bangladesh. 


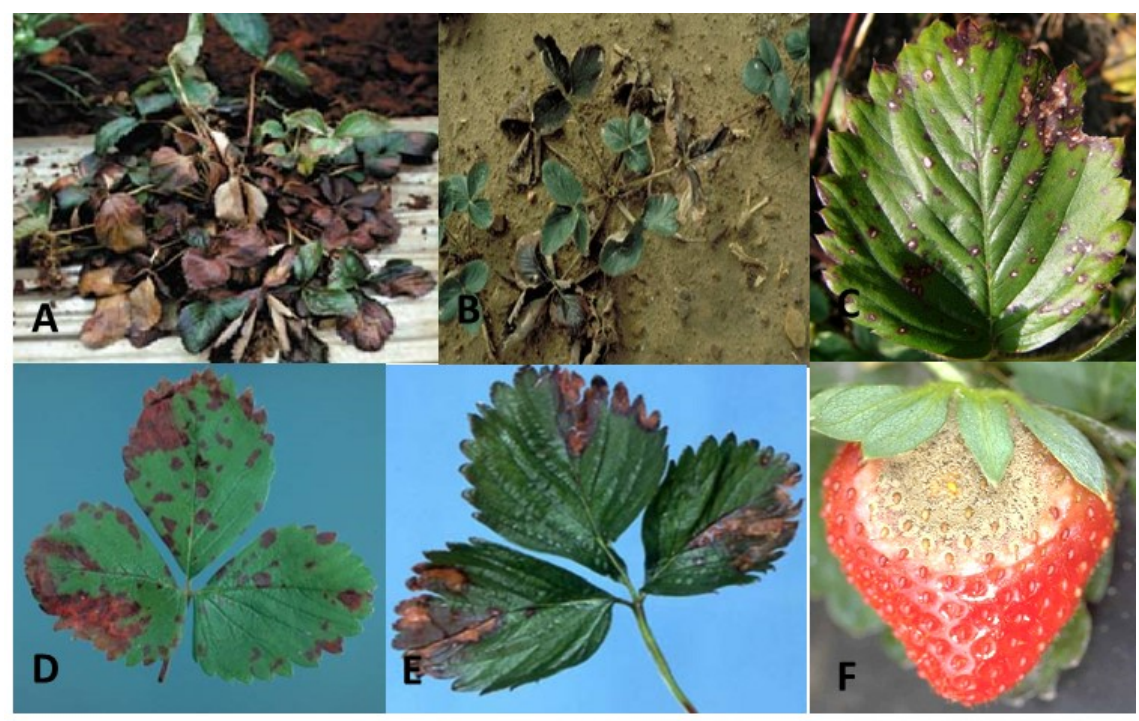

Fig. 3. Phytophthora crown rot disease of strawberry (A), strawberry plant dying from Verticillium wilt (B), strawberry leaf spot symptoms on leaflet (C), leaf scotch on strawberry (D), Pomopsis leaf blight on strawberry $(E)$ and Botrytis fruit rot on mature strawberry fruit $(F)$.

Table 5. Disease incidence (\%) of seven strawberry cultivars and their somaclones. Data were recorded after 90-120 days after plantation in the field.

\begin{tabular}{lcccccc}
\hline \multirow{2}{*}{$\begin{array}{l}\text { Cultivers/ } \\
\text { Somaclones }\end{array}$} & \multicolumn{5}{c}{ Disease incidence (\%) } \\
\cline { 2 - 6 } & Verticillium wilt & $\begin{array}{c}\text { Phytophthora crown } \\
\text { rot }\end{array}$ & Leaf scotch & Leaf spot & Leaf blight & $\begin{array}{c}\text { Botrytis } \\
\text { fruit rot }\end{array}$ \\
\hline AOG & 60 & 60 & 50 & 50 & 50 & 45 \\
AOG SC 1 & 15 & 10 & 10 & 10 & 10 & 10 \\
AOG SC 2 & 15 & 15 & 10 & 10 & 10 & 10 \\
AOG SC 3 & 10 & 10 & 00 & 00 & 00 & 00 \\
AOG SC 4 & 15 & 15 & 10 & 10 & 10 & 10 \\
\hline JP-2 & 60 & 60 & 50 & 50 & 50 & 50 \\
JP-2 SC 1 & 15 & 15 & 10 & 10 & 10 & 10 \\
JP-2 SC 2 & 15 & 15 & 10 & 10 & 10 & 10 \\
\hline JP-3 & 60 & 60 & 50 & 50 & 50 & 50 \\
JP-3 SC 1 & 15 & 15 & 10 & 10 & 10 & 10 \\
JP-3 SC 2 & 15 & 15 & 10 & 10 & 10 & 10 \\
\hline
\end{tabular}


Table 5 Contd.

\begin{tabular}{lllllll}
\hline Camarosa & 60 & 60 & 50 & 50 & 50 & 50 \\
Camarosa SC 1 & 15 & 15 & 10 & 10 & 10 & 10 \\
Camarosa SC 2 & 15 & 15 & 10 & 10 & 10 & 10 \\
\hline Sweet Charly & 60 & 60 & 50 & 50 & 50 & 50 \\
Sweet Charly SC 1 & 15 & 15 & 10 & 10 & 10 & 10 \\
Sweet Charly SC 2 & 15 & 15 & 10 & 10 & 10 & 10 \\
\hline Giant Mountain & 60 & 60 & 50 & 50 & 50 & 50 \\
Giant Mountain SC 1 & 15 & 15 & 10 & 10 & 10 & 10 \\
Giant Mountain SC 2 & 15 & 15 & 10 & 10 & 10 & 10 \\
\hline Festival & 60 & 60 & 50 & 50 & 50 & 50 \\
Festival SC 1 & 15 & 15 & 10 & 10 & 10 & 10 \\
Festival SC 2 & 15 & 15 & 10 & 10 & 10 & 10 \\
\hline
\end{tabular}

Table 6. Summer overcoming potentiality of seven strawberry varieties and their somaclones. Data were recorded 120 days after plantation in the field.

\begin{tabular}{|c|c|c|c|}
\hline \multirow[b]{2}{*}{ Varieties/Somaclones } & \multicolumn{3}{|c|}{ Summer overcoming potentiality (\%) } \\
\hline & $\begin{array}{c}\text { Low } \\
\left(28-30^{\circ} \mathrm{C}\right)\end{array}$ & $\begin{array}{l}\text { Moderate } \\
\left(30-35^{\circ} \mathrm{C}\right)\end{array}$ & $\begin{array}{c}\text { High } \\
\text { (above } 35^{\circ} \mathrm{C} \text { ) }\end{array}$ \\
\hline AOG & 95 & 5 & -- \\
\hline AOG SC 1 & 10 & 75 & 15 \\
\hline AOG SC 2 & 10 & 75 & 15 \\
\hline AOG SC 3 & -- & 80 & 20 \\
\hline AOG SC 4 & 10 & 75 & 15 \\
\hline JP-2 & 95 & 5 & -- \\
\hline JP-2 SC 1 & 10 & 75 & 15 \\
\hline JP-2 SC 2 & 10 & 75 & 15 \\
\hline JP-3 & 95 & 5 & -- \\
\hline JP-3 SC 1 & 10 & 75 & 15 \\
\hline JP-3 SC 2 & 10 & 75 & 15 \\
\hline Camarosa & 95 & 5 & -- \\
\hline Camarosa SC 1 & 10 & 75 & 15 \\
\hline Camarosa SC 2 & 10 & 75 & 15 \\
\hline Sweet Charly & 95 & 5 & -- \\
\hline Sweet Charly SC 1 & 10 & 75 & 15 \\
\hline Sweet Charly SC 2 & 10 & 75 & 15 \\
\hline Giant Mountain & 95 & 5 & -- \\
\hline Giant Mountain SC 1 & 10 & 75 & 15 \\
\hline Giant Mountain SC 2 & 10 & 75 & 15 \\
\hline Festival & 95 & 5 & -- \\
\hline Festival SC 1 & 10 & 75 & 15 \\
\hline Festival SC 2 & 10 & 75 & 15 \\
\hline
\end{tabular}




\section{Acknowledgement}

The authors thankfully acknowledge the Ministry of Science and Technology (MOST) of the Peoples Republic of Bangladesh for financial support for this study.

\section{References}

Abbas KH, Al- Sallihy AAM and Inrahim KM (2017). Contamination free callus cultures in strawberry (Fragaria $\times$ ananassa Dutch cv.), International Journal of Chemtech Research 10(9): 633-640.

Ara T, Karim MR, Aziz MA, Karim R, Islam R and Hossain M (2013). Micropropagation and field evaluation of seven strawberry genotypes suitable for agro-climatic condition of Bangladesh, African Journal of Agricultural Research 8(13): 1194-1199.

Boxus P (1974). The production of strawberry plants by in vitro micro-propagation, Journal of Horticultural Science 49: 209-210.

Finstad K and Martin RR (1995). Transformation of strawberry for virus resistance, Acta Horticulturae 385: 86-90.

Goffreda JC, Scopel AL and Fiola JA (1995). Indole butyric acid induces regeneration of phenotypically normal apricot (Prunus armeniaca L.) plants from immature embryos, Plant Growth Regulation 17: 41-46.

Haque MM (1997). Genetic study of yield components under soil moisture stress condition in chickpea (Cicer arietinum L.). M Sc Thesis, Department of Botany, Rajshahi University, Bangladesh.

Hossain MM (2007). Strawberry Cultivation In Bangladesh (Bangla). Sarker Publications, 38, Bangla Bazar, Dhaka-1100.

Jain SM, Brar DS and Ahloowalia BS (1998). Somaclonal variation and induced mutations in crop improvement, Kluwer, London.

Jelenkovic G, Chin C, Billings S and Eberhardt J (1991). Transformation studies in cultivated strawberry, Fragaria $\times$ ananassa Duch. In: "The Strawberry into the $21^{\text {st }}$ Century", (Eds.): Dale A and Lubby JJ, Timber Press, Portland, Oregon. 91-97 pp.

Jimenez-Bermudez S and Redondo- Nevado J (2002). Manipulation of strawberry fruit softening by antisense expression of a Pectate Lyase gene. Plant Physiology 128: 751 - 759.

Jones OP, Waller BJ and Beech MG (1988). The production of strawberry plants from callus cultures, Plant Cell, Tissue and Organ Culture 12: 235-2 41.

Karim R, Ahmed F, Roy UK, Ara T, Islam R and Hossain M (2015). Varietal improvement of strawberry (Fragaria $\times$ ananassa Dutch.) through somaclonal variation using in vitro techniques, Journal of Agricultural Science and Technology 17(4): 977-986.

Larkin PJ and Scowcroft WR (1981). Somaclonal variation - a novel source of variability from cell cultures for plant improvement, Theoretical and Applied Genetics 60: 197-214.

Liu ZR and Sanford JC (1988). Plant regeneration by organogenesis from strawberry leaf and runner tissue, HortScience 23: 1057-1059.

Mahmoud $\mathrm{O}$ and Kosar M (2013). Regeneration and histological of plants derived from leaf explants in vitro culture of strawberry, International Journal of Agriculture and Crop Sciences 5(9): 943-950.

Murashige, T. and Skoog F. 1962. A revised medium for rapid growth and bioassays with tobacco cultures. Physiologia Plantarum 15: 473- 497.

Nasrin S, Hossain MM, Khatun A, Alam MF and Mondal MRK (2003). Induction and evaluation of somaclonal variation in potato (Solanum tuberosum L.), Journal of Biological Sciences 3: 183-190. 
Nehra NS and Stushnoff C (1989). Direct shoot regeneration from strawberry leaf disks, Journal of the American Society for Horticultural Science 114(6): 1014-1018.

Nehra NS, Stushnoff C and Kartha KK (1990). Regeneration of plants from immature leaf-derived callus of strawberry (Fragaria $\times$ ananassa), Plant Science 66(1): 119-126.

Owen HR and Miller AR (1996). Haploid plant regeneration from anther cultures of three North American cultivars of strawberry (Fragaria $\times$ ananassa Duch), Plant Cell Reports 15: 905-909.

Passey AJ, Barrett KJ and James DJ (2003). Adventitious shoot regeneration from seven commercial strawberry cultivars (Fragaria $\times$ ananassa Duch.) using a range of explant types, Plant Cell Reports 21: 397-401.

Patnaik J, Sahoo S and Debata BK (1999). Somaclonal variation in cell suspension culture-derived regenerants of Cymbopogon martinii (Roxb.) wats var. Motia, Plant Breeding 118: 351-354.

Popescu AN, Isac VS, Coman MS and Radulescu MS (1997). Somaclonal variation in plants regenerated by organogenesis from callus culture of strawberry (Fragaria × ananassa), Acta Horticulturae 439: 89-95.

Rahman MW, Zohora S, Talukder MAI and Kayess MO (2015). Effect of different hormone combinations on callus induction and plant regeneration of strawberry, International Journal of Advanced Research 3(6): 1244-1250.

Rieger M (2006). Introduction to fruit crops. The Haworth Press, Binghamton, New York, 145 pp. retrieved from http://www.fruit-crops.com/strawberry- Fragaria- $x$-ananassa/. 